\title{
High Age Could Influence Large Thrombus Original Aspiration Catheter Advancement over the Carotid Siphon
}

Yoshihisa Matsumoto, ${ }^{1}$ Hayatsura Hanada, ${ }^{2}$ Kouhei Nii, ${ }^{2}$ Yusuke Morinaga, ${ }^{2}$ Minoru Iko, ${ }^{3}$ Takafumi Mitsutake, ${ }^{2}$ Ayumu Eto, ${ }^{2}$ Hiroshi Aikawa, ${ }^{3}$ Kiyoshi Kazekawa, ${ }^{3}$ and Masanori Tsutsumi ${ }^{2}$

Objective: A direct aspiration technique using a 5MAXACE catheter (Penumbra, Alameda, CA, USA) has been reported. However, recanalization has not been achieved in all cases with this technique alone. Additionally, Japan has a rapidly aging society, and differences in the condition of vessels, because of aging, can limit the approach of revascularization devices to the thrombus. We evaluated the accessibility of the 5MAX ACE (0.060-inch inner diameter) over the clinoid segment of the internal carotid artery (ICA).

Methods: We conducted a retrospective and cross-sectional study of 28 patients who received intraarterial treatment for acute ischemic stroke between October 2014 and October 2016. We recorded the maximum distal arrival point of the distal edge of the 5MAX ACE during the procedure before the stent retriever was retrieved.

Results: In 5 of the 28 patients, the distal edge of the 5MAX ACE catheter did not advance over the clinoid segment of the ICA. The mean age of patients for whom the 5MAX ACE catheter failed to advance over the clinoid segment of the ICA was significantly higher (non-advancement: $85 \pm 3$ years) than that of patients with advancement (76 \pm 9 years, Mann-Whitney U-test: $\mathrm{P}=0.04$ ).

Conclusion: Advanced age may limit successful revascularization using only a direct aspiration technique with the 5MAX ACE without a stent or balloon anchoring technique for lesions at the terminal portion of the ICA and more distal arteries, which suggests that different devices or approaches may be needed for clot removal.

Keywords $5 \mathrm{MAX}$ ACE, high-aged population, accessibility, clinoid segment of the internal carotid artery

\section{Introduction}

Intravenous recombinant tissue-type plasminogen activator (rt-PA) is recommended for eligible patients and significantly improves the overall odds of a good stroke outcome when delivered within 4.5 hours of stroke onset. ${ }^{1,2)}$ However,

${ }^{1}$ Department of Neurosurgery, Tanushimaru Central Hospital, Kurume, Fukuoka, Japan

${ }^{2}$ Department of Neurosurgery, Fukuoka University Chikushi Hospital, Chikushi, Fukuoka, Japan

${ }^{3}$ Department of Neurosurgery, Fukuoka Neurosurgical Hospital, Fukuoka, Fukuoka, Japan

Received: April 3, 2018; Accepted: September 21, 2018

Corresponding author: Yoshihisa Matsumoto. Department of Neurosurgery, Tanushimaru Central Hospital, 892 Masuoda, Tanushimaru, Kurume, Fukuoka 839-1213, Japan

Email: fwip4873@mb.infoweb.ne.jp

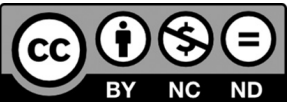

This work is licensed under a Creative Commons Attribution-NonCommercialNoDerivatives International License.

(C)2019 The Japanese Society for Neuroendovascular Therapy intravenous rt-PA has limitations. ${ }^{2)}$ Christou et al. ${ }^{3)}$ reported that after receiving intravenous rt-PA, $67 \%$ of middle cerebral artery (MCA) occlusions, $25 \%$ of basilar artery (BA) occlusions, and all internal carotid artery (ICA) occlusions were not completely recanalized. To overcome the limitations of rt-PA and obtain better outcomes, further interventions have been considered. In patients with acute ischemic stroke caused by proximal intracranial occlusion of the anterior circulation, intraarterial treatment administered within 6 hours after stroke onset was found to be effective and safe. ${ }^{4)}$

The development of new revascularization devices has improved recanalization rates and time. Thrombectomy with a stent retriever improved the functional outcomes. ${ }^{5)}$ A direct aspiration first pass technique (ADAPT) with a large bore aspiration catheter as the first approach for thrombectomy has been reported. ${ }^{6}$ The largest caliber aspiration catheter that the vessel could accommodate was selected for each case, usually a 5MAX (0.054-inch inner diameter; Penumbra, Alameda, CA, USA) or 5MAX ACE 
(0.060-inch inner diameter; Penumbra), for distal ICA, proximal MCA, and BA occlusions. The large bore aspiration catheter is placed at the face of the thrombus, and aspiration is carried out. ${ }^{7)}$ The utility of the combined aspiration and stent retrieval technique for thrombectomy has also been reported. ${ }^{8-10)}$

In the ADAPT FAST study, large bore aspiration catheters easily navigated in the intracranial circulation through a guide catheter (Neuron 088; Penumbra, Oakland, CA, USA). Successful revascularization by aspiration technique alone was achieved in $78 \%$ of cases. ${ }^{7)}$ However, delivering the large aspiration catheter to the target vessel and advancement through the ophthalmic segment of the ICA may present as a challenge, ${ }^{11,12)}$ especially with very acute angle. ${ }^{13)}$ Delayed angiographic reperfusion decreased likelihood of good clinical outcome. ${ }^{14)}$ Rapid recanalization could be achieved only if the device could be quickly navigated to the occluded vessel. When too much forward force is used to deliver the catheter, the parent vessel is at risk for injury, and the catheter may be damaged. ${ }^{13)}$ To overcome the difficulty of delivering the large aspiration catheter over the carotid siphon, the utility of the "grappling hook" technique using the Merci retriever system ${ }^{13}$ ) and stent retriever ${ }^{9)}$ as an anchoring system was reported. We considered that if it is difficult to deliver the large aspiration catheter to the target vessel and to aspirate the clot, quick transition to other techniques may be needed to achieve fast and safe recanalization.

It is also not possible to achieve successful revascularization anytime using a stent retriever alone. There are patients who were needed aspiration technique with a large bore aspiration catheter. In the contact aspiration vs Stent Retriever for Successful Revascularization (ASTER) study, successful revascularization was achieved in $67.7 \%$ of the patients after first-line stent retriever thrombectomy alone. Therefore, rescue treatment, which included contact aspiration, combined contact aspiration and stent retriever, and angioplasty, was performed in $23.8 \%$ of the patients after stent retriever thrombectomy in the first-line stent retriever group. ${ }^{15)}$

Advanced age is associated with complex anatomical risk factors including internal carotid tortuosity ${ }^{16)}$ and difficult catheter access to the target carotid artery during endovascular treatment for acute ischemic stroke. ${ }^{17)}$ Japan has a rapidly aging society. In a report involving consecutive patients with stroke treated with rt-PA in 10 Japanese stroke centers, the age of patients was $72 \pm 12$ years, ${ }^{18)}$ which was higher than that of the large studies that provided evidence on endovascular therapy for acute ischemic stroke. The mean age of the patients was $65.0 \pm 12.5$ years in the stent retriever plus intravenous rt-PA group of Solitaire with the Intention for Thrombectomy as Primary Endovascular Treatment for Acute Ischemic Stroke (SWIFT PRIME), ${ }^{5)} 65.7 \pm 11.3$ years in the thrombectomy group of the Randomized Trial of Revascularization With Solitaire FR Device versus Best Medical Therapy in the Treatment of Acute Stroke Due to Anterior Circulation Large Vessel Occlusion Presenting within Eight Hours of Symptom Onset (REVASCAT) trial, ${ }^{19)} 66.3$ years in the ADAPT FAST study, ${ }^{7}$ and $68.6 \pm 12.3$ years in the endovascular therapy group of Extending the Time for Thrombolysis in Emergency Neurological Deficits - Intra-Arterial (EXTEND-IA). ${ }^{20)}$ The median age was 65.8 years in the intervention group of the Multicenter Randomized Clinical Trial of Endovascular Treatment for Acute Ischemic Stroke in the Netherlands (MR CLEAN) and 71 years in the intervention arm of the Endovascular Treatment for Small Core and Anterior Circulation Proximal Occlusion with Emphasis on Minimizing CT to Recanalization Times (ESCAPE) trial. ${ }^{4,21)}$

If the predictive factors that make it difficult to recanalize with aspiration using 5MAX ACE alone are identified, the other devices and treatments could be selected promptly to achieve fast and safe recanalization. Therefore, to assess the association between successful 5MAX ACE catheter advancement over the carotid siphon and various patient characteristics, the present study evaluated the results of intraarterial treatment of acute ischemic stroke.

\section{Materials and Methods}

In Japan, the 5MAX ACE catheter became available for use for intracranial vessels in October 2014. We retrospectively collected data from the medical records of patients with acute ischemic stroke caused by a proximal intracranial arterial occlusion and who received intraarterial treatment at the Fukuoka University Chikushi Hospital and Tanushimaru Central Hospital between October 2014 and October 2016. As this study's design was retrospective and cross-sectional, the treatments, including the procedure, were not performed as part of this study, but as part of patient management. This study was approved by the ethics committee of the Fukuoka University Medical Ethics Review Board (FU-MERB) (Permission number: R16-053) and carried out according to the guidelines of the committee. This study was also approved by the ethics 
committee of the Tanushimaru Central Hospital (Permission number: 2017-1) and carried out according to the guidelines of the committee.

The endovascular approach consisted of aspiration or stent retrieval alone or a combination of revascularization devices. The selection of the device and approach was based on the discretion of each doctor. To evaluate the ability of the 5MAX ACE catheter advancement, we included the patients for whom the 5MAX ACE (0.060-inch inner diameter) was used for intracranial anterior circulation lesions.

During the procedure, a guide catheter was advanced distally as far as possible into the cervical or proximal petrous ICA. The 5MAX ACE catheter was advanced usually coaxially over a 0.014-inch guidewire and Marksman microcatheter (ev3, Irvine, CA, USA) or other obturating catheters, such as 3MAX (Penumbra) or Trevo Pro 18 Microcatheter (Stryker, Kalamazoo, MI, USA). We advanced the inner catheter of the 5MAX ACE through the thrombus. We advanced the 5MAX ACE as distally as possible and/or tried to directly aspirate the thrombus. If the 5MAX ACE could not achieve adequate revascularization or to reach the thrombus, we used techniques combined with other revascularization devices. We applied aspiration through the distal aspiration catheter and/or proximal flow arrest using a balloon-guided catheter for protection against new embolisms. We did not use the balloon-inflation anchoring technique or change the inner catheter to $3 \mathrm{MAX}$ during the procedure for more distal advancement of the 5MAX ACE.

To assess the efficacy of 5MAX ACE, we recorded the maximum distal arrival point of the distal edge of the 5MAX ACE during the procedure before the stent retriever was retrieved. Because the stent could be used as anchoring system for distal advancement of the microcatheter, ${ }^{9,22,23)}$ the 5MAX ACE moved distally when the stent retriever was pulled. We evaluated the ability of the 5MAX ACE to advance over the carotid siphon without a stent anchoring technique by checking whether the distal edge of the 5MAX ACE could advance over the clinoid segment of the ICA. ${ }^{24)}$

\section{Study definitions and evaluation techniques}

The medical records were dependent on patient selfreporting, but the final records were left to the discretion of physician after a review of the patients' self-reports and in-hospital examination results.

Angiography of the anteroposterior and lateral projections was routinely performed during the procedure to evaluate intracranial blood flow. We assessed cavernous
ICA (cICA) tortuosity using the classification written by Lin et al. ${ }^{25)}$ The open configurations/angles of the genus are defined as type I. The closed configuration of the anterior genu is defined as type II. Type II has more acute angle of the genu than type I. The buckled appearance at the posterior deflection of the posterior genu is defined as type III. The posterior genu is buckled superiorly than the anterior genu like a shape of the Simmons-type catheter defined as type IV. The type I, types II-III, and type IV are classified with mild, moderate, and severe tortuosity. ${ }^{25}$ ) These assessments of tortuosity were assessed by personnel who were blinded to information regarding the treatment. Revascularization was measured using the modified thrombolysis in cerebral infarction (mTICI) score as recommended by the cerebral angiographic revascularization grading collaborators. ${ }^{26)}$ Successful revascularization was defined as an mTICI score $\geq 2 \mathrm{~b}$ post-treatment. The procedure time was defined as the time from groin access to at least $\mathrm{mTICI} 2 \mathrm{~b}$ revascularization.

\section{Statistical analysis}

The Mann-Whitney U-test was used to determine significant differences in age, and procedure time. Fisher's exact test was used to assess all other parameters. In this study, the cICA tortuosity was divided into two groups, minimal group or moderate to severe group. The data are presented as the mean \pm standard deviation. Statistical significance was accepted if the probability level was less than 0.05. All statistical tests were two-tailed.

\section{Results}

Figure 1 shows the study flow chart. We retrospectively enrolled 57 patients (58 cases) who underwent endovascular procedures for acute ischemic stroke during the 25 -month period. We excluded 30 cases from this study, including 3 cases treated at the ICA origin alone, 9 cases treated at the posterior circulation, 1 case for whom 5MAX instead of 5MAX ACE was used, and 17 cases treated using other revascularization devices (e.g., stent retriever) without 5MAX ACE. Thus, we finally evaluated 28 patients ( 28 cases, 12 men and 16 women). The age of the study group was $78 \pm 9$ years (mean \pm standard deviation, median: 79 , range: $60-94)$. In these 28 patients, occlusion of the terminal portion of the ICA and/or MCA (M1 or M2) was detected during the procedure.

In 8 of the 28 patients, a stiff-type guidewire and/or Simmons-type coaxial catheter was needed to deploy the 


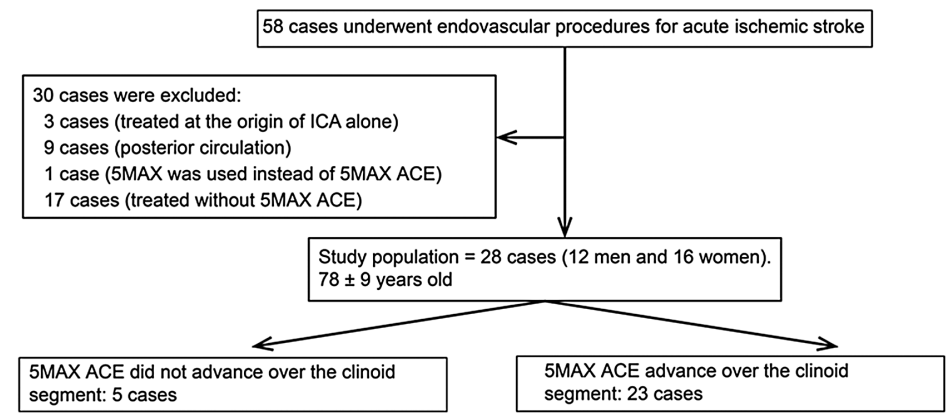

Fig. 1 Study flow chart. ICA: internal carotid artery

guide catheter. The balloon-guided catheter was used in 17 patients. A Marksman microcatheter was used in 26 of the 28 patients, 3MAX was used in 1 patient, and a Trevo Pro 18 Microcatheter was used in 1 patient with 5MAX ACE.

In 5 of the 28 patients, the maximum distal arrival point of the distal edge of the 5MAX ACE did not advance over the clinoid segment of ICA. In these five patients, the guide catheter was advanced as distally as possible into the ICA.

In the group in which the 5MAX ACE advanced over the clinoid segment, 13 of 23 patients had a right-side lesion. About the cICA tortuosity classification, minimal (type I) type was detected in 16 patients, and moderate (types II-III) in 7 patients. Occlusion of the terminal portion of the ICA was detected in 11 patients. Nine patients had M1 and 3 had M2. The balloon-guided catheter was used in 15 patients. The inner catheter was advanced to M1 in 2 patients and M2 or a more distal artery in 21 patients. Successful revascularization was achieved in 17 of the 23 patients. One lesion at the ICA and 5 lesions at the M1 achieved successful revascularization only by the direct aspiration technique using the 5MAX ACE. The other 11 cases achieved successful revascularization via a combined technique with other revascularization devices. During the procedure, 7 of the 23 patients were evaluated collateral circulation flow before the revascularization procedure. The length of the procedure was $78 \pm 35$ minutes.

In the group in which the 5MAX ACE did not advance over the clinoid segment, 2 of 5 patients had a right-side lesion. About the cICA tortuosity classification, minimal (type I) type was detected in three patients, moderate (types II-III) in one patient, and severe or Simmons-type (type IV) in one patient. Occlusion of the terminal portion of the ICA was detected in two patients. Two of the patients had M1 and 1 had M2. The balloon-guided catheter was used in two patients. The inner catheter was advanced to M2 or a more distal artery in all patients. Successful revascularization occurred in three of the five patients. None of these three patients achieved successful revascularization by only the direct aspiration technique using the 5MAX ACE. These three cases achieved successful revascularization using a combined technique with other revascularization devices. During the procedure, three of the five patients were evaluated collateral circulation flow before the revascularization procedure. The length of the procedure was $92 \pm 36$ minutes.

We compared several indices between the groups in which the distal edge of the 5MAX ACE did not advance over the clinoid segment of the ICA (five cases) and the rest of the patient population (23 cases). The mean age of patients for whom the $5 \mathrm{MAX}$ ACE did not advance over the clinoid segment of the ICA was significantly higher (advancement over the clinoid segment group: $76 \pm 9$ years, no advancement group: $85 \pm 3$ years, Mann-Whitney $\mathrm{U}$-test: $\mathrm{P}=0.04)$. There were no statistically significant differences between these groups regarding other parameters (Table 1). Additionally, the mean age of patients that required the stiff-type guidewire and/or Simmons-type coaxial catheter was $84 \pm 8$ years, whereas the mean age for those who did not need these devices was $75 \pm 8$ years. There was a statistically significant difference between both groups (Mann-Whitney U-test; $\mathrm{P}=0.02$ ).

\section{Representative cases}

\section{Case 1: A 70-year-old man (Fig. 2)}

The patient lived at home without assistance. He experienced sudden right hemiparesis and aphasia and was diagnosed with cerebral infarction, which was treated with intravenous rt-PA and endovascular therapy. The angle between the aortic arch to the left common carotid artery (CCA) was not acute and it was easy to deploy the guide catheter at the ICA. DSA showed MCA occlusion and the 
Table 1 Comparison of patients according to 5MAX ACE advancement over the clinoid segment

\begin{tabular}{|c|c|c|c|}
\hline & $\begin{array}{l}\text { Advancement over } \\
\text { clinoid segment }\end{array}$ & No advancement & $P$ value \\
\hline $\mathrm{N}$ & 23 & 5 & \\
\hline Gender (men), n (\%) & $10(43)$ & $2(40)$ & 1 \\
\hline Age $(y), n \pm S D$ & $76 \pm 9$ & $85 \pm 3$ & $0.04^{*}$ \\
\hline Hypertension, n (\%) & $8(35)$ & $3(60)$ & 0.3 \\
\hline Diabetes mellitus, $\mathrm{n}(\%)$ & $2(9)$ & $0(0)$ & 1 \\
\hline Dyslipidemia, n (\%) & $4(17)$ & $1(20)$ & 1 \\
\hline History of ischemic stroke event, $\mathrm{n}(\%)$ & $4(17)$ & $1(20)$ & 1 \\
\hline Right side lesion, n (\%) & $13(57)$ & $2(40)$ & 0.6 \\
\hline \multicolumn{4}{|l|}{ clCA tortuosity classification, $\mathrm{n}(\%)$} \\
\hline Minimal & $16(70)$ & $3(60)$ & \\
\hline Moderate-severe & $7(30)$ & $2(40)$ & 0.6 \\
\hline ICA occlusion, n (\%) & $11(48)$ & $2(40)$ & 1 \\
\hline M2 occlusion, n (\%) & $3(13)$ & $1(20)$ & 1 \\
\hline Balloon guide catheter, $\mathrm{n}(\%)$ & $15(65)$ & $2(40)$ & 0.3 \\
\hline Stiff guidewire or Simmonds coaxial catheter used, $\mathrm{n}(\%)$ & $5(22)$ & $3(60)$ & 0.1 \\
\hline Inner catheter was 3MAX, n (\%) & $1(4)$ & $0(0)$ & 1 \\
\hline Inner catheter was Marksman, $\mathrm{n}(\%)$ & $21(91)$ & $5(100)$ & 1 \\
\hline \multicolumn{4}{|l|}{ Inner catheter advancement, n (\%) } \\
\hline M1 & $2(9)$ & $0(0)$ & \\
\hline M2 or more distal artery & $21(91)$ & $5(100)$ & 1 \\
\hline Successful revascularization rate (mTICl 2b-3) n (\%) & 17 (74) & $3(60)$ & 0.6 \\
\hline Procedure time (min.) $n \pm S D$ & $78 \pm 35$ & $92 \pm 36$ & 0.3 \\
\hline
\end{tabular}

The Mann-Whitney U-test was used to determine significant differences for age and procedure time; Fisher's exact test was used for assessing all other parameters. ${ }^{\star} \mathrm{P}<0.05$ indicates statistical significance. cICA: cavernous internal carotid artery; ICA: internal carotid artery; mTICl: modified thrombolysis in cerebral infarction; SD: standard deviation
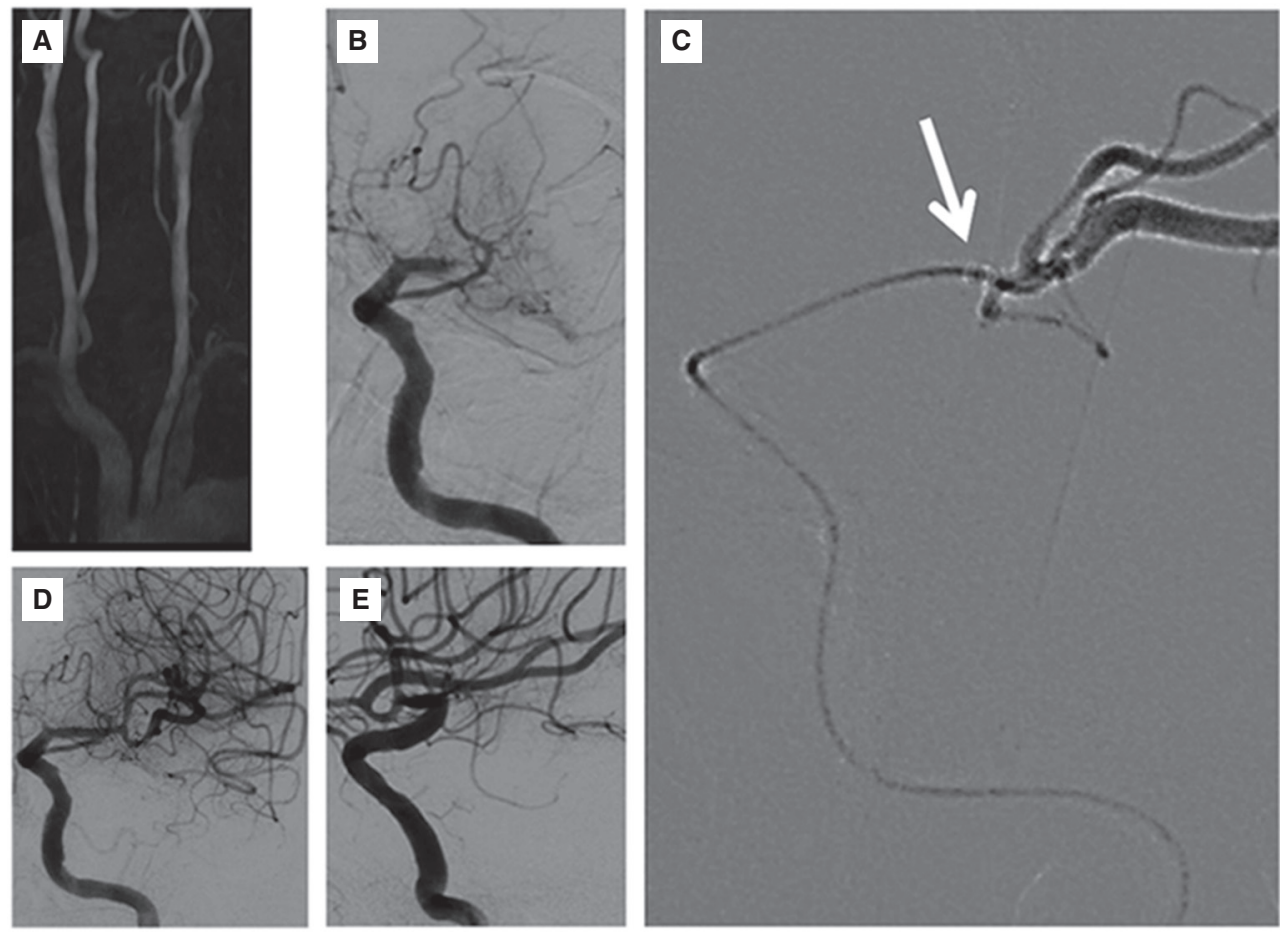

Fig. 2 Case 1. (A) MRA at the aortic arch to the ICA. (B) DSA (anteroposterior view) showing occlusion of the MCA. (C) DSA (left anterior oblique view) showing the deployment of the distal edge of the 5MAX ACE (arrow) at the MCA. (D) DSA (anteroposterior view) showing revascularization of the MCA after aspiration of the clot using 5MAX ACE. (E) DSA (lateral view) showing type I cavernous ICA. ICA: internal carotid artery; MCA: middle cerebral artery 

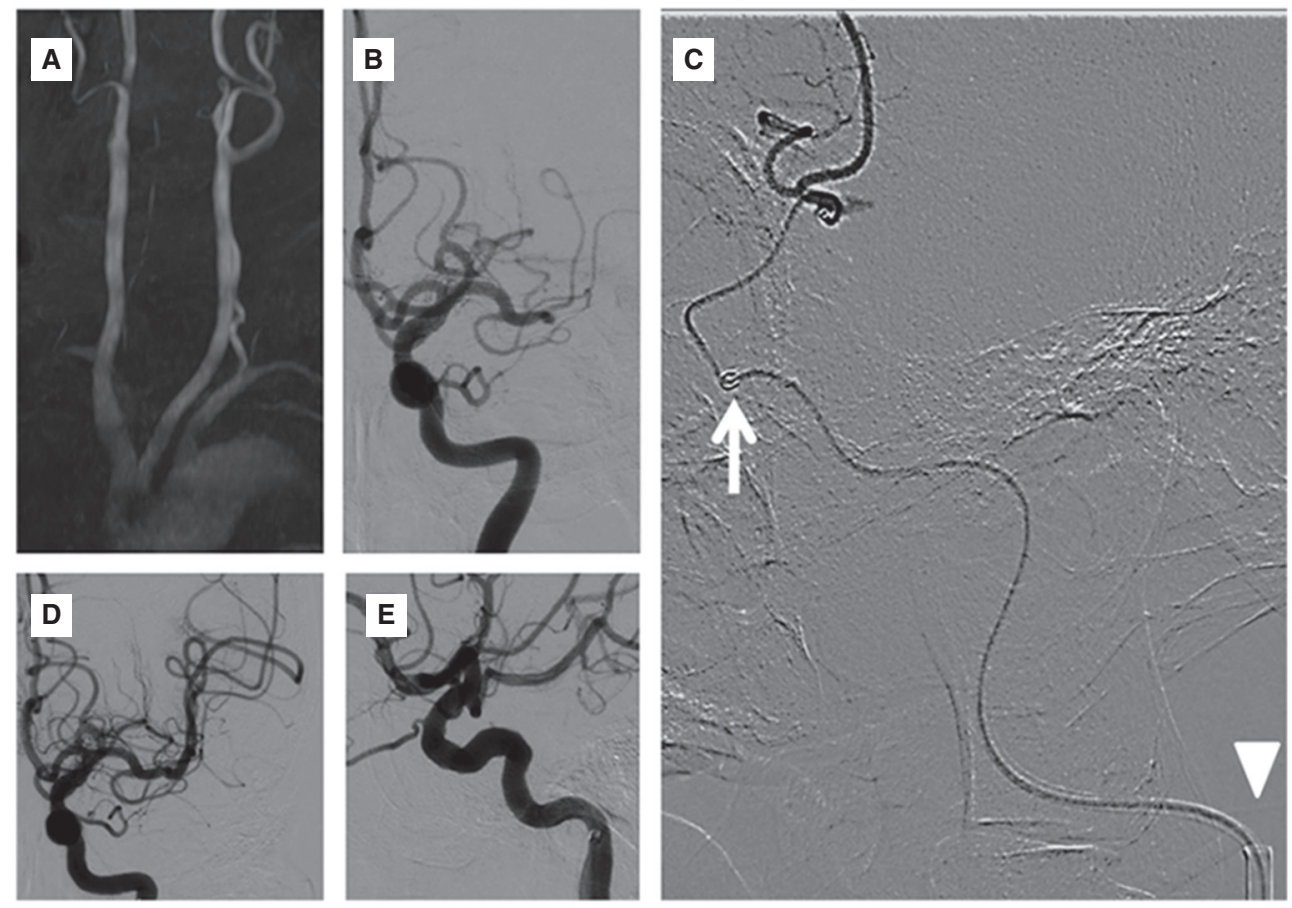

Fig. 3 Case 2. (A) MRA at the aortic arch to the ICA. The angle between the aortic arch and the left CCA was relatively acute. (B) DSA (anteroposterior view) showing occlusion of the MCA. (C) DSA (left anterior oblique view) showing that the distal edge of the 5MAX ACE (arrow) did not advance over the clinoid segment of the ICA. When we attempted to advance the 5MAX ACE, the guide catheter (arrowhead) could not maintain its position and was forcibly moved proximally and the 5MAX ACE could not be advanced any further. (D) DSA (anteroposterior view) showing revascularization of the MCA using the stent retriever. (E) DSA (lateral view) showing type III cavernous ICA. CCA: common carotid artery; ICA: internal carotid artery; MCA: middle cerebral artery

5MAX ACE was advanced to the thrombus in the MCA with Marksman microcatheter as the inner catheter. MCA revascularization was achieved after aspiration of the clot using 5MAX ACE. The cICA tortuosity was type I. The patient's symptoms were mild, and he was discharged home without assistance.

\section{Case 2: A 90-year-old woman (Fig. 3)}

The patient lived at home without assistance. She experienced sudden right hemiparesis and aphasia and was diagnosed with cerebral infarction. She underwent intravenous rt-PA and endovascular therapy. The angle between the aortic arch and the left CCA was found to be acute. It was difficult to deploy the guide catheter at the ICA using a normal guidewire, so a stiff-type guidewire was used instead. DSA showed occlusion of the MCA. We used 5MAX ACE with Marksman microcatheter as the inner catheter. The 5MAX ACE could not advance further over the clinoid segment of the ICA. The guide catheter could not maintain its position and was forcibly moved proximally when the SMAX ACE was advanced beyond this point. MCA revascularization was achieved using a stent retriever. The cICA tortuosity was type III. The patient's symptoms were mild, and she was discharged home without assistance.

\section{Discussion}

In this study, we evaluated the accessibility of 5MAX ACE catheter over the carotid siphon. The results of this study indicate that the mean age of patients for whom the SMAX ACE did not advance over carotid siphon was significantly higher than that of patients for whom advancement occurred.

The mean age of patients in the present study was $78 \pm 9$ years and was higher than that in previous studies that reported the effectiveness of intraarterial treatment for acute ischemic stroke. The mean or median age of patients in previously published large studies that provided evidence on endovascular therapy for acute ischemic stroke ranged from 65 to 71 years. $4,5,7,19-21)$ The exact reason for the higher mean age of patients in the 5MAX ACE no-advancement 
group is unknown. Age-associated changes occur in the arterial system over time and aging is associated with structural and functional changes in the arterial wall. ${ }^{27)}$ Aging may influence the ability of the 5MAX ACE to advance over the carotid siphon.

In cases where the 5MAX ACE did not advance over the carotid siphon, when we attempted to advance it beyond this point, the resistance was high, the guide catheter could not maintain its position, and it was forcibly moved proximally. These results suggest that the stability of the guide catheter is important for distal advancement of the 5MAX ACE. The anatomy of the aortic arch and cervical artery may influence the stability of the guide catheter. The patients $\geq 80$ years had an increased incidence of complex anatomic risk factors, which include arch calcification, common carotid/innominate stenosis, common carotid tortuosity, and internal carotid tortuosity, compared to younger patients. ${ }^{16)}$ An age $>75$ years was a predictor of difficult catheter access to the target carotid artery in acute stroke patients. ${ }^{17)}$ In the present study, we did not routinely obtain and evaluate the images between the aortic arch and cervical segment of the ICA for the endovascular treatment in all patients because of the time limitation for the treatment of ischemic stroke. We could not obtain the postoperative angiographic evaluation for the vascular angle and vessel diameter in all patients. However, when a stiff-type guidewire and/or Simmons-type coaxial catheter was used, it was difficult to deploy the guide catheter to the target vessel via the usual method. This suggested the tortuosity of the aortic arch and carotid artery. The stiff guidewire was useful for overcoming the tortuous vessels for the treatment of intracranial lesions. ${ }^{28)}$ In this study, the age of patients requiring the stiff-type guidewire and/or Simmonstype coaxial catheter was higher than that of patients who did not need these instruments.

There was a trend, but no statistically significant relationship between 5MAX ACE advancement over carotid siphon and the need for the stiff-type guidewire and/or Simmons-type coaxial catheter. In this study, the stiff-type guidewire and/or Simmons-type coaxial catheter was used in only 8 out of the 28 patients. A small number of cases may influence the statistical power. We will continue our evaluation with the larger number of cases whether the need for the stiff-type guidewire and/or Simmons-type coaxial catheter could influence the accessibility of the 5MAX ACE catheter over the carotid siphon.

With regard to the tortuosity of the carotid siphon and endovascular treatment, the classification of cICA tortuosity correlated strongly with markers of the pipeline embolization device (Covidien Vascular Therapies, Mansfield, MA, USA) procedural complexity. ${ }^{25}$ ) The acuteness of the carotid siphon angle influences the interference between two microcatheters for the jailing technique using the Neuroform EZ stent (Stryker) ${ }^{29)}$ In the treatment of acute ischemic stroke, the tortuous ophthalmic segment of ICA could restrict the accessibility of the large caliber aspiration catheters and make it necessary to apply adjunctive techniques. ${ }^{13)}$ In this study, the cICA tortuosity did not significantly influence the results. However, severe or Simmons-type (type IV) cICA tortuosity was found in only one patient and the 5MAX ACE did not advance over the clinoid segment in this patient. The present study involved a small number of cases. A statistically significant difference may be detected if the number of cases increases.

\section{Limitations}

One of the limitations of this study is the retrospective and cross-sectional design. Moreover, it involved a small number of cases, and it is possible that the statistical power of the study was insufficient. The subjective view of each doctor may have been reflected in device selection, and this selection bias may have affected the study results.

We did not use the balloon-inflation anchoring technique to advance the 5MAX ACE. The inner catheter could be navigated to the peripheral artery with severe vessel tortuosity by dilating/anchoring a balloon of the balloon-guided catheter. ${ }^{30)}$ This technique could advance the 5MAX ACE more distally. The large aspiration catheter required a coaxial catheter for delivery to MCA. ${ }^{12)}$ In the present study, a Marksman microcatheter was used in most cases instead of a 3MAX as the inner catheter of 5MAX ACE. 3MAX is commonly used to support catheterization with the ACE/ACE 64 reperfusion catheters. ${ }^{31}$ Although the utility of the coaxial technique with a 0.021inch microcatheter (Prowler select plus; Cordis, Miami, FL, USA) with a large bore catheter has been reported, ${ }^{11)}$ the $3 \mathrm{MAX}$ as the inner catheter could advance the 5MAX ACE more distally than the Marksman microcatheter. Recently, the utility of combined treatment with a balloonguided catheter, a large bore aspiration catheter for distal aspiration, and a stent retriever has been reported. ${ }^{10)}$ The utility of the Penumbra ACE and 3MAX reperfusion system for M2 and M3 occlusions in acute ischemic stroke has also been reported. ${ }^{31)}$ In the present study, we evaluated the ability of the 5MAX ACE to advance over the 
carotid siphon without a stent anchoring technique. The usefulness of a stent anchoring technique with a distal aspiration catheter to overcome the tortuous anatomy has been reported.9) We will continue our investigation with anchoring techniques and $3 \mathrm{MAX}$.

In this study, we evaluated relatively few anatomical parameters. For example, we did not evaluate the calcification of intracranial ICA by CT because the imaging conditions were not consistent in our two institutions. Although the calcification on the intracranial carotid artery does not have an impact on reperfusion or clinical outcomes in acute ischemic stroke undergoing endovascular therapy, ${ }^{32)}$ analyzing further anatomical parameters may find the factors related to better catheter selections. Because of these limitations, a prospective, randomized trial with a larger patient population focused on better catheter selections is needed.

\section{Conclusion}

Increased age may limit revascularization only by a direct aspiration technique by $5 \mathrm{MAX}$ ACE without anchoring techniques for lesions at the terminal portion of the ICA and more distal artery and suggest the use of different devices or approaches for clot removal. We will seek a more appropriate approach for elderly patients and assess the new generation devices, including ACE 68 (Penumbra).

\section{Acknowledgments}

We are grateful to Yukiko Nakano, Hosei Eto, Kimiya Sakamoto, Ritsuro Inoue, Yasuyuki Nomoto, Kousuke Kumagai, Hitoshi Maeda, Fumihiro Hiraoka, and Shuko Hamaguchi for their help with this study. We would like to thank Editage (www.editage.com) for English language editing.

\section{Disclosure Statement}

There is no conflict of interest for the first author and coauthors.

\section{References}

1) Emberson J, Lees KR, Lyden P, et al: Effect of treatment delay, age, and stroke severity on the effects of intravenous thrombolysis with alteplase for acute ischaemic stroke: a meta-analysis of individual patient data from randomised trials. Lancet 2014; 384: 1929-1935.

2) Jauch EC, Saver JL, Adams HP, et al: Guidelines for the early management of patients with acute ischemic stroke: a guideline for healthcare professionals from the American Heart Association/American Stroke Association. Stroke 2013; 44: 870-947.

3) Christou I, Burgin WS, Alexandrov AV, et al: Arterial status after intravenous TPA therapy for ischaemic stroke. A need for further interventions. Int Angiol 2001; 20: 208-213.

4) Berkhemer OA, Fransen PS, Beumer D, et al: A randomized trial of intraarterial treatment for acute ischemic stroke. $N$ Engl J Med 2015; 372: 11-20.

5) Saver JL, Goyal M, Bonafe A, et al: Stent-retriever thrombectomy after intravenous t-PA vs. t-PA alone in stroke. $N$ Engl J Med 2015; 372: 2285-2295.

6) Turk AS, Spiotta A, Frei D, et al: Initial clinical experience with the ADAPT technique: a direct aspiration first pass technique for stroke thrombectomy. J Neurointerv Surg 2014; 6: 231-237.

7) Turk AS, Frei D, Fiorella D, et al: ADAPT FAST study: a direct aspiration first pass technique for acute stroke thrombectomy. J Neurointerv Surg 2014; 6: 260-264.

8) Humphries W, Hoit D, Doss VT, et al: Distal aspiration with retrievable stent assisted thrombectomy for the treatment of acute ischemic stroke. J Neurointery Surg 2015; 7: 90-94.

9) McTaggart RA, Tung EL, Yaghi S, et al: Continuous aspiration prior to intracranial vascular embolectomy (CAPTIVE): a technique which improves outcomes. J Neurointerv Surg 2017; 9: 1154-1159.

10) Maegerlein $C$, Mönch S, Boeckh-Behrens $T$, et al: PROTECT: proximal balloon occlusion together with direct thrombus aspiration during stent retriever thrombectomy evaluation of a double embolic protection approach in endovascular stroke treatment. J Neurointery Surg 2017; 10: 751-755.

11) Lee HC, Kang DH, Hwang YH, et al: Forced arterial suction thrombectomy using distal access catheter in acute ischemic stroke. Neurointervention 2017; 12: 45-49.

12) Spiotta AM, Chaudry MI, Hui FK, et al: Evolution of thrombectomy approaches and devices for acute stroke: a technical review. J Neurointerv Surg 2015; 7: 2-7.

13) Hui FK, Hussain MS, Spiotta A, et al: Merci retrievers as access adjuncts for reperfusion catheters: the grappling hook technique. Neurosurgery 2012; 70: 456-460.

14) Khatri $P$, Yeatts SD, Mazighi M, et al: Time to angiographic reperfusion and clinical outcome after acute ischaemic stroke: an analysis of data from the Interventional Management of Stroke (IMS III) phase 3 trial. Lancet Neurol 2014; 13: 567-574.

15) Lapergue B, Blanc R, Gory B, et al: Effect of endovascular contact aspiration vs stent retriever on revascularization in patients with acute ischemic stroke and large vessel occlusion: the ASTER randomized clinical trial. JAMA 2017; 318 : 443-452.

16) Lin SC, Trocciola SM, Rhee J, et al: Analysis of anatomic factors and age in patients undergoing carotid angioplasty and stenting. Ann Vasc Surg 2005; 19: 798-804. 
17) Ribo M, Flores A, Rubiera M, et al: Difficult catheter access to the occluded vessel during endovascular treatment of acute ischemic stroke is associated with worse clinical outcome. J Neurointerv Surg 2013; 5 Suppl 1: i70-i73.

18) Toyoda K, Koga M, Naganuma M, et al: Routine use of intravenous low-dose recombinant tissue plasminogen activator in Japanese patients: general outcomes and prognostic factors from the SAMURAI register. Stroke 2009; 40: 3591-3595.

19) Jovin TG, Chamorro A, Cobo E, et al: Thrombectomy within 8 hours after symptom onset in ischemic stroke. $N$ Engl $J$ Med 2015; 372: 2296-2306.

20) Campbell BC, Mitchell PJ, Kleinig TJ, et al: Endovascular therapy for ischemic stroke with perfusion-imaging selection. N Engl J Med 2015; 372: 1009-1018.

21) Goyal M, Demchuk AM, Menon BK, et al: Randomized assessment of rapid endovascular treatment of ischemic stroke. N Engl J Med 2015; 372: 1019-1030.

22) Fargen KM, Velat GJ, Lawson MF, et al: The stent anchor technique for distal access through a large or giant aneurysm. J Neurointerv Surg 2013; 5: e24.

23) Kan P, Wakhloo AK, Mokin M, et al: Techniques in distal access of wide-necked giant intracranial aneurysms during treatment with flow diversion. Surg Neurol Int 2015; 6: S284-S288.

24) Bouthillier A, Van Loveren HR, Keller JT: Segments of the internal carotid artery: a new classification. Neurosurgery 1996; 38: 425-433.
25) Lin LM, Colby GP, Jiang B, et al: Classification of cavernous internal carotid artery tortuosity: a predictor of procedural complexity in pipeline embolization. J Neurointerv Surg 2015; 7: 628-633.

26) Zaidat OO, Yoo AJ, Khatri P, et al: Recommendations on angiographic revascularization grading standards for acute ischemic stroke: a consensus statement. Stroke 2013; 44: 2650-2663.

27) Strait JB, Lakatta EG: Aging-associated cardiovascular changes and their relationship to heart failure. Heart Fail Clin 2012; 8: 143-164.

28) Eckard DA, Krehbiel KA, Johnson PL, et al: Stiff guide technique: technical report and illustrative case. AJNR Am J Neuroradiol 2003; 24: 275-278.

29) Yoon SG, Jin SC, Kim SH, et al: Jailing technique using a catheter-based open-cell stent system in internal carotid artery sidewall aneurysms unfeasible to simple coiling. $J$ Cerebrovasc Endovasc Neurosurg 2013; 15: 293-298.

30) Tokunaga S, Tsurusaki Y, Sambongi Y, et al: Balloon- inflation anchoring technique for insertion of a guiding catheter in acute mechanical thrombectomy. JNET 2017; 11: 53-58.

31) Altenbernd J, Kuhnt O, Hennigs S, et al: Frontline ADAPT therapy to treat patients with symptomatic M2 and M3 occlusions in acute ischemic stroke: initial experience with the Penumbra ACE and 3MAX reperfusion system. J Neurointerv Surg 2018; 10: 434-439.

32) Haussen DC, Gaynor BG, Johnson JN, et al: Carotid siphon calcification impact on revascularization and outcome in stroke intervention. Clin Neurol Neurosurg 2014; 120: 73-77. 